\title{
Composite Lymphoma: Opposite Ends of Spectrum Meet
}

\author{
Uqba Khan ${ }^{\text {a, b }}$, Tarik Hadid ${ }^{\mathrm{a}}$, Warda Ibrar ${ }^{\mathrm{a}}$, Dahlia Sano ${ }^{\mathrm{a}}$, \\ Ayad Al-Katib
}

\begin{abstract}
An 18-year-old African-American female presented with an episode of syncope. Initial investigations revealed large lung mass with invasion into right atrium along with lesions in kidneys and liver. Patient also developed superior vena cava syndrome due to lung mass. Biopsy of lung mass revealed diagnosis of composite lymphoma with involvement by primary mediastinal B-cell lymphoma (PMBCL) and classical Hodgkin lymphoma. Patient was started on dose-adjusted etoposide, prednisone, vincristine, cyclophosphamide, doxorubicin and rituximab (EPOCH-R) with complete response to treatment. This case represents an extremely rare type of aggressive lymphoma and can guide clinicians in managing such cases since there are no standard guidelines for treatment. To the best of our knowledge, this is the first reported case of composite lymphoma of PMBCL and classical Hodgkin lymphoma successfully treated with dose-adjusted EPOCH$\mathrm{R}$ regimen.
\end{abstract}

Keywords: Composite lymphoma; Primary mediastinal B-cell lymphoma; EPOCH-R

\section{Introduction}

Lymphomas are the most distinct and diverse group of malignancies that are classified into various subcategories according to clinical and pathological features. Lymphoma is mainly divided into Hodgkin lymphoma and non-Hodgkin lymphoma; however, each of these is sub-divided into various types depending on morphology, cell of origin, cytogenetic, molecular and clinical features. Despite major advancements in therapeutic options for lymphomas, there are many challenges as well, including management of composite lymphoma. Composite lymphoma is defined as a type of lymphoma in which there is synchronous presence of two distinct varieties of lymphoma in a single patient [1]. Composite lymphomas can be composed

Manuscript accepted for publication January 17, 2017

aSt. John Hospital and Medical Center, Detroit, MI, USA

${ }^{b}$ Corresponding Author: Uqba Khan, 19229 Mack Ave., Suite 23, Grosse

Pointe Woods, Detroit, MI 48236, USA. Email: uqba.md@gmail.com

doi: https://doi.org/10.14740/jocmr2929w of two different types of non-Hodgkin lymphoma or synchronous presence of Hodgkin and non-Hodgkin lymphoma in a single patient [2]. Composite lymphoma comprised of primary mediastinal B-cell lymphoma (PMBCL) and Hodgkin lymphoma is extremely rare and only a handful number of cases have been reported in literature [3]. We describe a unique case of composite lymphoma with component of PMBCL and classical Hodgkin lymphoma. This case represents an extremely rare type of aggressive lymphoma and can guide clinicians in managing such cases since there are no standard guidelines for treatment.

\section{Case Report}

An 18-year-old African-American female came to emergency room after an episode of syncope. She reported progressive swelling of her neck and face for 3 weeks. Her past medical, surgical and family history was unremarkable. She was not taking any medication prior to this hospitalization. She denied smoking tobacco, alcohol abuse or recreational drug use. Physical examination was significant for generalized swelling of neck and face. There was no lymphadenopathy, hepatomegaly or splenomegaly appreciated on examination.

Laboratory evaluation revealed WBC of $10,400 / \mathrm{mm}^{3}$, hemoglobin of $9.4 \mathrm{~g} / \mathrm{dL}$, platelets of $378,000 / \mathrm{mm}^{3}$, creatinine of $0.58 \mathrm{mg} / \mathrm{dL}$ and lactate dehydrogenase of $368 \mathrm{IU} / \mathrm{L}$ (upper limit of normal: $240 \mathrm{IU} / \mathrm{L})$. CT scan showed a $14 \times 12 \times$ $10 \mathrm{~cm}$ mass in right lung along with liver lesion and multiple small masses in both kidneys. Echocardiogram revealed large, spherical, fixed mass in the right atrial cavity. CT-guided core needle biopsy of lung mass revealed the diagnosis of composite lymphoma with components of PMBCL and focal Hodgkin lymphoma. Needle core biopsies showed two different morphologic processes, and majority of the cores showed an infiltrate of large atypical cells associated with clear cytoplasm and fine fibrosing compartmentalization. Second area of the needle cores showed an infiltrate composed of small lymphocytes, neutrophils and eosinophils with scattered large atypical cells. Immunohistochemical stains also revealed two different patterns; the large atypical cells in the large cell infiltrate were positive for B-cell markers (CD20 and CD79a) and CD23, and negative for CD10 and showed weak expression for bcl-6 and bcl-2. Ki-67 in this subset was 70\%. The other subset of cells was positive for CD15 and CD30 with negative to weak ex- 

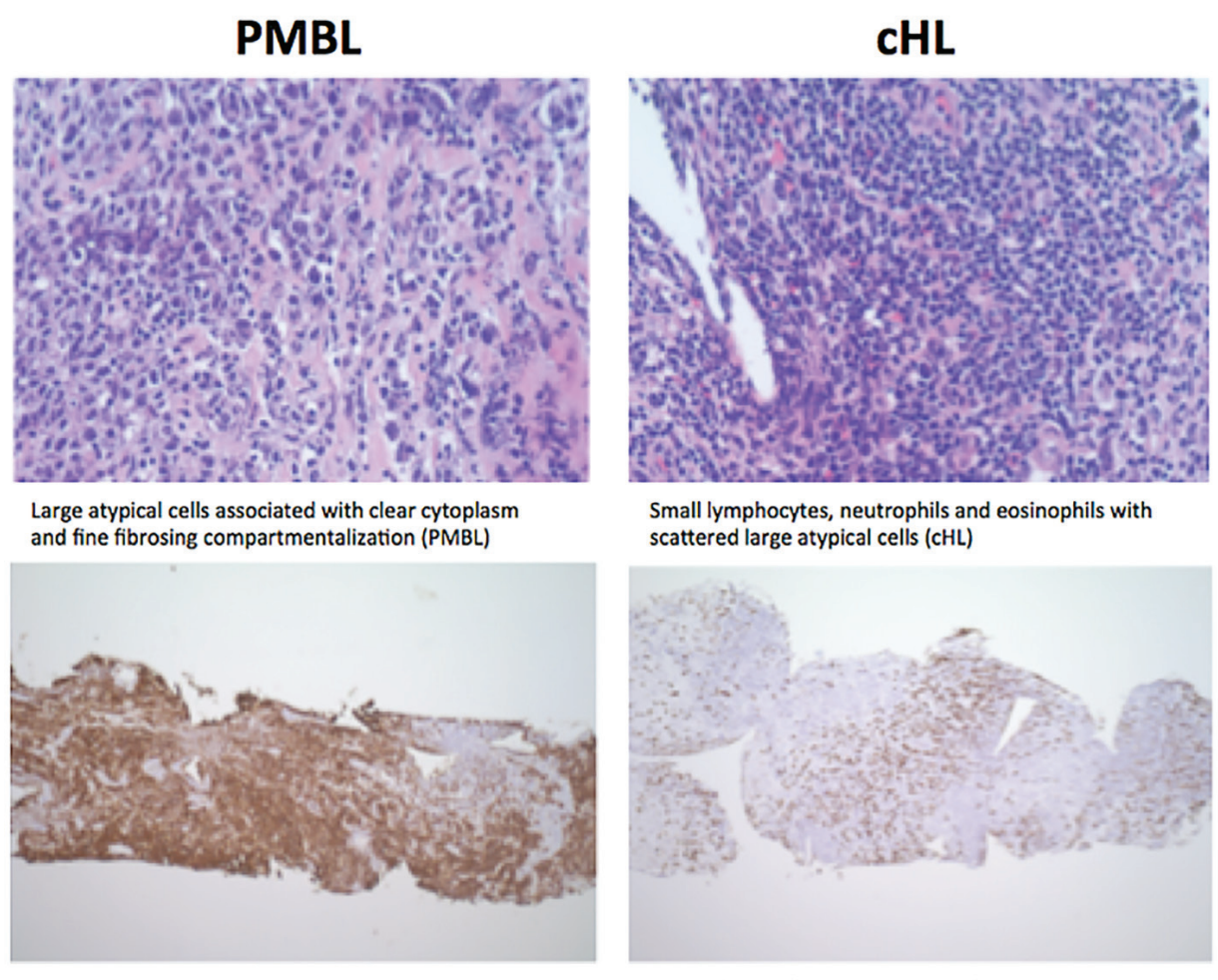

Small lymphocytes, neutrophils and eosinophils with scattered large atypical cells (cHL)

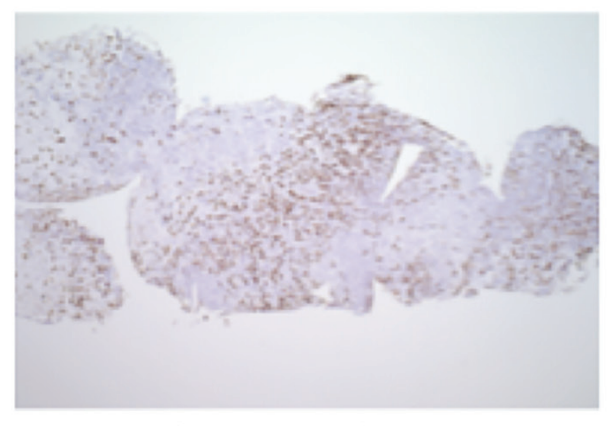

CD20 highlighting PMBL

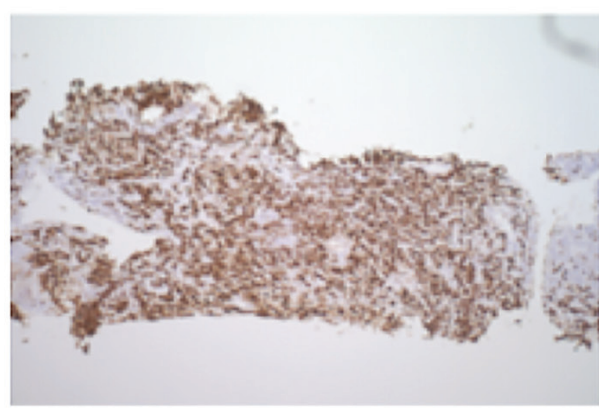

$\mathrm{Ki}-67$ expression in the large B-cell infiltrate is estimated at $70 \%$

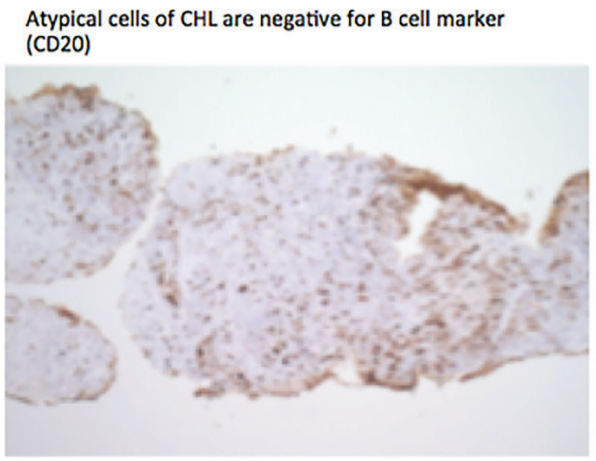

$\mathrm{Ki}-67$ highlights scattered large cells only, estimated at 10-15\%.

Figure 1. Pathological comparison of PMBCL and Hodgkin lymphoma on biopsy specimen.

pression of PAX-5 (Fig. 1). Bone marrow biopsy showed no involvement with lymphoma.

During her initial presentation, patient developed superior vena cava syndrome due to large lung mass and also found out to have pulmonary embolism. After establishing the diagnosis, patient was started on dose-adjusted etoposide, prednisone, vincristine, cyclophosphamide, doxorubicin and rituximab (EPOCH-R) chemotherapy. Patient showed excellent clinical response to treatment and after just two cycles of therapy, her superior vena cava syndrome resolved completely. She was also given CNS prophylaxis with intrathecal methotrexate from third cycle onwards. After six cycles of treatment, PET scan showed no evidence of disease. Patient received a total of eight cycles of treatment with dose-adjusted EPOCH$\mathrm{R}$. Patient remains in complete remission after completion of treatment.

\section{Discussion}

Our case represents a unique and rare entity of lymphoma called composite lymphoma. Composite lymphoma is defined as a type of lymphoma in which there is synchronous presence of two distinct varieties of lymphoma in a single patient [1].

Composite lymphoma comprising of PMBCL and Hodgkin lymphoma is very rare. PMBCL usually presents in third and fourth decades of life and affects adolescents and young adults. PMBCL is typically confined in mediastinum; however, disseminated disease may occur at diagnosis with involvement of extranodal sites such as liver, kidney and adrenal gland [4]. 
Morphologically, it comprises of medium to large cells having round or lobulated nuclei and abundant cytoplasm [4]. PMBCL expresses B cell-associated antigens including CD19, CD20, CD22 and CD79a [5]. The germinal center markers are usually expressed in most cases of PMBCL $[6,7]$.

PMBCL shares many clinical, morphological and genetic features with nodular sclerosis Hodgkin lymphoma. Clinically, both of them can present with mediastinal mass and are more common in young females. In terms of morphological features, occasionally, some neoplastic cells are multinucleated and resemble Hodgkin-Reed-Sternberg cells [8]. Gene expression profiling studies reveal many genotype similarities between PMBCL and nodular sclerosis Hodgkin lymphoma [9, 10]. Amplification of the REL proto-oncogene and the JAK2 tyrosine kinase gene are frequently seen in both entities $[11,12]$.

Management of composite lymphoma is extremely challenging, as there is no known standard of care treatment. Dose-adjusted EPOCH-R has been used successfully in patients with PMBCL with overall survival rate of $97 \%$ at median of 5-year follow-up [13]. This regimen has active drugs against Hodgkin lymphoma including etoposide, vincristine, cyclophosphamide, prednisone and doxorubicin. In essence, the only active drug in treatment of Hodgkin lymphoma missing from EPOCH-R is bleomycin. We also incorporated CNS prophylaxis with intrathecal methotrexate due to increased risk of CNS involvement, as there were multiple organs involved with lymphoma in our patient.

Composite lymphoma presents a major clinical challenge for clinicians, as there are no established guidelines available. Our case highlights that EPOCH-R appears to be effective and appropriate choice for composite lymphoma of PMBCL and classical Hodgkin lymphoma. However, continuing follow-up is necessary in our case to determine the long-term outcome of this treatment.

\section{References}

1. Kim H, Hendrickson R, Dorfman RF. Composite lymphoma. Cancer. 1977;40(3):959-976.

2. Kuppers R, Duhrsen U, Hansmann ML. Pathogenesis, diagnosis, and treatment of composite lymphomas. Lancet Oncol. 2014;15(10):e435-446.

3. Traverse-Glehen A, Pittaluga S, Gaulard P, Sorbara L, Alonso MA, Raffeld M, Jaffe ES. Mediastinal gray zone lymphoma: the missing link between classic Hodgkin's lymphoma and mediastinal large B-cell lymphoma. Am J Surg Pathol. 2005;29(11):1411-1421.

4. Dunleavy K, Wilson WH. Primary mediastinal B-cell lymphoma and mediastinal gray zone lymphoma: do they require a unique therapeutic approach? Blood. 2015;125(1):33-39.

5. Lamarre L, Jacobson JO, Aisenberg AC, Harris NL. Primary large cell lymphoma of the mediastinum. A histologic and immunophenotypic study of 29 cases. Am J Surg Pathol. 1989;13(9):730-739.

6. Calaminici M, Piper K, Lee AM, Norton AJ. CD23 expression in mediastinal large B-cell lymphomas. Histopathology. 2004;45(6):619-624.

7. Salama ME, Rajan Mariappan M, Inamdar K, Tripp SR, Perkins SL. The value of CD23 expression as an additional marker in distinguishing mediastinal (thymic) large B-cell lymphoma from Hodgkin lymphoma. Int J Surg Pathol. 2010;18(2):121-128.

8. Steidl C, Gascoyne RD. The molecular pathogenesis of primary mediastinal large B-cell lymphoma. Blood. 2011;118(10):2659-2669.

9. Rosenwald A, Wright G, Leroy K, Yu X, Gaulard P, Gascoyne RD, Chan WC, et al. Molecular diagnosis of primary mediastinal B cell lymphoma identifies a clinically favorable subgroup of diffuse large B cell lymphoma related to Hodgkin lymphoma. J Exp Med. 2003;198(6):851862 .

10. Savage KJ, Monti S, Kutok JL, Cattoretti G, Neuberg D, De Leval L, Kurtin P, et al. The molecular signature of mediastinal large B-cell lymphoma differs from that of other diffuse large B-cell lymphomas and shares features with classical Hodgkin lymphoma. Blood. 2003;102(12):3871-3879.

11. Joos S, Kupper M, Ohl S, von Bonin F, Mechtersheimer G, Bentz M, Marynen P, et al. Genomic imbalances including amplification of the tyrosine kinase gene JAK2 in CD30+ Hodgkin cells. Cancer Res. 2000;60(3):549-552.

12. Joos S, Menz CK, Wrobel G, Siebert R, Gesk S, Ohl S, Mechtersheimer G, et al. Classical Hodgkin lymphoma is characterized by recurrent copy number gains of the short arm of chromosome 2. Blood. 2002;99(4):1381-1387.

13. Dunleavy K, Pittaluga S, Maeda LS, Advani R, Chen CC, Hessler J, Steinberg SM, et al. Dose-adjusted EPOCHrituximab therapy in primary mediastinal B-cell lymphoma. N Engl J Med. 2013;368(15):1408-1416. 